\title{
Prevalence of DSM-IV Major Psychiatric Disorders among North Korean Defectors in South Korea
}

\author{
Kyoung Eun Lee', Carolyn Seungyoun Moon', Ji Hyun An¹, Hyo Chul Lee', Da Eun Kim¹, \\ Subin Park'2 ${ }^{2}$ Minji Lee ${ }^{2}$, Hye In Chang $^{3}$, and Jin Pyo Hong ${ }^{1}$ \\ ${ }^{1}$ Department of Psychiatry, Sungkyunkwan University School of Medicine, Samsung Medical Center, Seoul, Republic of Korea \\ 2Department of Research Planning, Mental Health Research Institute, National Center for Mental Health, Seoul, Republic of Korea \\ ${ }^{3}$ Department of Psychology, Sungkyunkwan University, Seoul, Republic of Korea
}

\begin{abstract}
Objective The aim of this study was to estimate the prevalence of major psychiatric disorders among North Korean defectors (NKD) settled in South Korea.

Methods The study population consisted of 294 North Korean defectors, aged 18 to 64 years settled in South Korea for 3 years or less. Between June 1, 2016 and October 31, 2016, face-to-face interviews were conducted using the North Korean version of the WHO-Composite International Diagnostic Interview (NK-CIDI) to diagnose DSM-IV psychiatric disorders.

Results The lifetime prevalence of any DSM-IV psychiatric disorders was $62.2 \%$ in NKD and $25.0 \%$ in the general Korean population. The prevalence of specific disorders in NKD and general Korean population was as follows: $22.3 \%$ and $4.9 \%$ for major depressive disorder (MDD), $12.2 \%$ and $1.4 \%$ for post-traumatic stress disorder (PTSD), $18.0 \%$ and $4.8 \%$ for nicotine dependence, and $14.5 \%$ and $11.2 \%$ for alcohol abuse. The incidence of every single psychiatric disorder varied in each country. For instance, the generalized anxiety disorder, specific phobia, and alcohol use disorder occurred more frequently in North Korea whereas PTSD was more prevalent in other countries. Conclusion The prevalence of psychiatric disorders among NKD was quite higher than in the general population of South Korea.
\end{abstract}

Psychiatry Investig 2020;17(6):541-546

Key Words North Korean defectors, Prevalence, Mental disorder, Mental health.

\section{INTRODUCTION}

Due to the economic and diplomatic crisis, poverty, food shortage, several North Korean defectors escaped from their homeland and decided to settle down in South Korea. ${ }^{1}$ The number of North Korean defectors who have settled in South Korea has increased continuously since 2005. According to the data released by the Unification Department, it reached 30,805 as of the end of June 2017. ${ }^{2}$ As the number of North Korean defectors increases, helping their successful adaptation to South Korean society has become a major social challenge in South Korea. $^{3}$

Received: August 20, 2019 Revised: December 14, 2019

Accepted: March 8, 2020

$\triangle$ Correspondence: Jin Pyo Hong, MD, PhD

Department of Psychiatry, Sungkyunkwan University School of Medicine, Samsung Medical Center, 81 Irwon-ro, Gangnam-gu, Seoul 06351, Republic of Korea

Tel: +82-2-3410-3585, Fax: +82-2-3410-0050, E-mail: suhurhong@gmail.com (c) This is an Open Access article distributed under the terms of the Creative Commons Attribution Non-Commercial License (https://creativecommons.org/licenses/by$\mathrm{nc} / 4.0$ ) which permits unrestricted non-commercial use, distribution, and reproduction in any medium, provided the original work is properly cited.
Refugees could be at high risk of psychiatric morbidity because of political or social reasons underlying forced migration, traumatic events, and resettlement in totally new environments. ${ }^{4}$ Several studies in the past investigated the prevalence of serious mental disorder among refugees: one meta-analysis suggested that about $10 \%$ of refugees in Western countries manifest post-traumatic stress disorder, $5 \%$ exhibit major depression, and $4 \%$ have a generalized anxiety disorder. ${ }^{5}$ Similar to other refugees, North Korean defectors have experienced severe traumatic events, which hinder their successful adaptation into South Korean society. ${ }^{6,7}$ Some North Korean defectors cross the border into China. China has highly regard for its relationship with North Korea, and does not grant refugee status to North Korean defectors; instead, they are captured as illegal immigrants and returned to North Korea. Previous studies report that North Korean defectors experience various traumas such as trauma related to physical abuse, discovery and arrest, separation from family members, and betrayal even during the period between their defection and their safe arrival in South Korea. ${ }^{8}$ As a result, it has been re- 
ported that North Korean defectors had high prevalence and severity of psychiatric symptoms, depression, anxiety and post-traumatic stress disorder (PTSD) ${ }^{7,9,10}$

Few studies compared mental health among North Korean defectors and the general Korean population using the same evaluation scale. ${ }^{11-13}$ These studies reported a higher prevalence of psychiatric disorders among North Korean defectors. The prevalence of depression ranged from 33\% to 51\% using the Korean version of the Center for Epidemiologic Studies Depression Scale ${ }^{11,14-19}$ and the prevalence of PTSD ranged from $13 \%$ to $52 \%$ using the post-traumatic diagnostic scale. ${ }^{11,20-25}$ Studies have yet to evaluate the prevalence using a structured diagnostic tool such as Composite International Diagnostic Interview (CIDI). ${ }^{26}$

In South Korea, the Korean Epidemiologic Catchment Area (KECA) study has been performed every 5 years since 2001 using the Korean version of CIDI by the Korea Ministry of Health and Welfare to determine the prevalence of major mental disorders among Korean adults. ${ }^{25}$ The prevalence of major mental disorders was investigated among North Korean defectors using the North Korean version of CIDI (NKCIDI) compared with the results of 2016 KECA..$^{27}$

\section{METHODS}

\section{Participants and procedures}

This study was conducted between 1st June and 31st October 2016. Participants were selected from 8 regional resettlement centers for North Korean defectors (Hana Center) across the country. The study population consisted of 294 North Korean defectors aged 18 to 64 years within 3 years of settling in South Korea. Psychology, nursing, and social work graduate students, who had experience in psychiatric epidemiologic surveys, were recruited as interviewers. All interviewers received the WHO-recommended CIDI training and performed face-to-face interview after the training. This study was approved by the institutional review board of Samsung Medical Center (SMC 2015-05-042-008). All of the participants were fully informed about the aims and methods of the study and provided written informed consent before the interview.

\section{Diagnostic assessments}

The North Korean version of Composite International Diagnostic Interview 2.1 (NK-CIDI 2.1) was administered to all subjects by trained interviewers. The CIDI is one of the epidemiological research tools, which were suitable for international diagnostic epidemiological research. ${ }^{28}$ The CIDI is a fully structured diagnostic interview designed for psychiatric diagnosis according to the DSM-IV. The K-CIDI was validated by Cho et al. ${ }^{29}$ following the World Health Organization guide- lines. Although Korean is the national language of both South Korea and North Korea, communication between the people of the Koreas is becoming increasingly difficult as the peninsula continues to remain divided. North Korean defectors have difficulty in communication because they are unfamiliar with the definitions and usage of various words, especially those related to emotions, commonly used in South Korean society. ${ }^{30} \mathrm{NK}-\mathrm{CIDI}$ was developed by Lee et al. ${ }^{31}$ as part of North Korean language psychiatric evaluation tool. NK-CIDI is a tool for proof of validity and reliability of K-CIDI, which is appropriately translated and developed based on sociocultural backgrounds of North Korea. Lifetime DSM-IV disorders included mood disorders (major depressive disorder, dysthymia, and bipolar disorders), anxiety disorder (posttraumatic stress disorder, panic disorder, agoraphobia, social phobia, generalized anxiety disorder, and specific phobia), nicotine use disorder and alcohol use disorder (alcohol dependence and abuse).

\section{The 2016 epidemiological survey of mental disorders in Korea}

The 2016 KECA study ${ }^{27}$ is a nationally representative survey of the prevalence of mental disorders among Korean adults conducted in 2016 using the K-CIDI. ${ }^{29}$ Subjects were selected using a stratified, multi-stage clustered sample design based on a population census. Psychology, nursing, and social work graduate students, experienced in psychiatric epidemiologic surveys, received the WHO-recommended CIDI training and performed face-to-face interview. The $2016 \mathrm{KECA}$ study ${ }^{27}$ was approved by the institutional review board of Samsung Medical Center (No. 2015-05-042). Written informed consent was obtained from subjects prior to their participation in the study. Among 5,102 respondents who participated in faceto-face interviews, this analysis included 3,848 subjects aged between 18 and 64 years to compare the prevalence between South Koreans and North Korean defectors within the same age range.

\section{Statistical analysis}

Weighted scores were calculated for each participant to approximate the national population distributions of age and sex according to the 2015 Census. ${ }^{13,32}$ All statistical analyses were based on the weighted data. Weighted lifetime prevalence and $95 \%$ confidence intervals (CIs) were calculated using Exact (Clopper-Pearson) Confidence Limits formula. ${ }^{33}$ All statistical analyses were performed with statistical package for the social sciences (SPSS) version 23.0 (IBM Corp., Armonk, NY USA). 
Table 1. Sociodemographic characteristics of North Korean defectors $(\mathrm{N}=294)$ and Korean general population $(\mathrm{N}=3,848)$

\begin{tabular}{|c|c|c|}
\hline & $\begin{array}{l}\text { North Korean } \\
\text { defectors } \\
(\mathrm{N}=294)\end{array}$ & $\begin{array}{c}\text { Korean general } \\
\text { population } \\
(\mathrm{N}=3,848)\end{array}$ \\
\hline & $\mathrm{N}(\%)$ & $\mathrm{N}(\%)$ \\
\hline \multicolumn{3}{|l|}{ Sex } \\
\hline Female & $232(78.9)$ & $2,345(60.9)$ \\
\hline Male & $62(21.1)$ & $1,503(39.1)$ \\
\hline \multicolumn{3}{|l|}{ Age } \\
\hline $18-29$ & $84(28.6)$ & $759(19.7)$ \\
\hline $30-39$ & $76(25.9)$ & $788(20.5)$ \\
\hline $40-49$ & $80(27.2)$ & $1,028(26.7)$ \\
\hline $50-59$ & $49(16.6)$ & $893(23.2)$ \\
\hline$>60$ & $5(1.7)$ & $380(9.9)$ \\
\hline \multicolumn{3}{|l|}{ Marital status } \\
\hline Married & $115(39.2)$ & $2,453(63.7)$ \\
\hline Divorced/separated/widowed & $96(32.6)$ & $370(9.6)$ \\
\hline Unmarried & $83(28.2)$ & $1,023(26.6)$ \\
\hline \multicolumn{3}{|l|}{ Education } \\
\hline$\leq 9$ & $56(19.0)$ & $421(10.9)$ \\
\hline 10-12 (high school) & $179(60.9)$ & $1,219(31.7)$ \\
\hline$\geq 13$ (college or above) & $59(20.1)$ & $2,208(57.4)$ \\
\hline \multicolumn{3}{|l|}{ Monthly income } \\
\hline Less than 1,750 USD & $248(84.4)$ & $941(24.5)$ \\
\hline 1,750 USD or more & $46(15.6)$ & $2,903(75.5)$ \\
\hline
\end{tabular}

\section{RESULTS}

Table 1 presents the socio-demographic characteristics of the participants. A total of 294 subjects (62 males and $232 \mathrm{fe-}$ males) completed the interview. The proportion of females among these subjects is similar to that among actual North Korean defectors (71\%) as reported by the National Statistical Office. $^{2}$ The mean age was $41.63 \pm 12.6$ ranging from 18 to 64 years. Among those participants, 39.2\% were married, 32.6\% were divorced, separated, or widowed, and $28.2 \%$ were singles. For the completed education years, $19 \%$ had 9 or less years, $60.9 \%$ had 10 to 12 years (high school), and $20.1 \%$ had 13 or above years (college) of education and the mean of completed education years was11.2 \pm 2.4 years. Most of North Korean defectors (84.4\%) had a household income less than 1,750 USD/month.

Table 2 shows a lifetime prevalence of major DSM-IV disorder among North Korean defectors and Korean general population along with 95\% CIs. About half of North Korean (50.12\%) defectors had psychiatric disorder in their lifetime excluding nicotine and alcohol use disorder compared with
$12.25 \%$ of general Korean population. North Korea had a higher prevalence in most measured disorders. The biggest difference in prevalence was in panic disorder (12.17 times) followed by PTSD (8.91 times), generalized anxiety disorder (GAD) (6.48 times), agoraphobia (6.42 times), dysthymic disorder (4.69 times), and major depressive disorder (MDD) (4.58 times).

Table 3 displays the prevalence of psychiatric disorder among North Korean defectors, a third country, and among South Koreans. The onset areas of MDD and both nicotine withdrawal and dependence were relatively evenly distributed. Panic disorder, GAD, specific phobia, and both alcohol dependence and abuse occurred the most in North Korea. The onset of dysthymic disorder and PTSD was observed in a third country the most while agoraphobia originated the most in South Korea.

\section{DISCUSSION}

This study investigated the prevalence of major psychiatric disorders among North Korean defectors within 3 years of settling in South Korea and compared them with comparable data from the 2016 epidemiological survey of mental disorders in Korea. $^{27}$

As a result, the prevalence of major psychiatric disorders in North Korean defectors was much higher than in the Korean general population. While escaping from North Korea and staying long-term in the third country, North Korean defectors might have experienced traumatic events or forced repatriation, which was related to the relatively high prevalence of psychiatric disorder. ${ }^{9}$ Moreover, a study of refugees reported that the socio-political backgrounds of refugee experience were related to mental health. ${ }^{34}$

In case of MDD, the lifetime prevalence of North Korean defectors was $22.32 \%$ and was 4.58 times higher than in general Korean population. The relevant previous studies reported that $30 \%$ of North Korean defectors have clinical MDD when diagnosed using a CES-D cut-off score of $25 .{ }^{15}$ Moreover, when CES-D cut-off score of 21 was applied, $51.4 \%$ of North Korean defectors and $10.4 \%$ of the general South Korean population have MDD. ${ }^{17}$ Although marginally lower compared with self-reported scales such as CES-D, the result of this study is congruent with previous studies reporting that North Korean defectors show about 5 times higher prevalence of MDD compared with the general South Korean population.

In addition, for the onset area of MDD, North Korea accounted for $36.8 \%$, the third country showed $34.1 \%$, and South Korea had $29.1 \%$ each indicating a relatively similar rate suggesting that not only the process of escaping from North Korea but also settling down in South Korea influenced their devel- 
Table 2. Lifetime prevalence of major psychiatric disorder among North Korean defectors and Korean general population

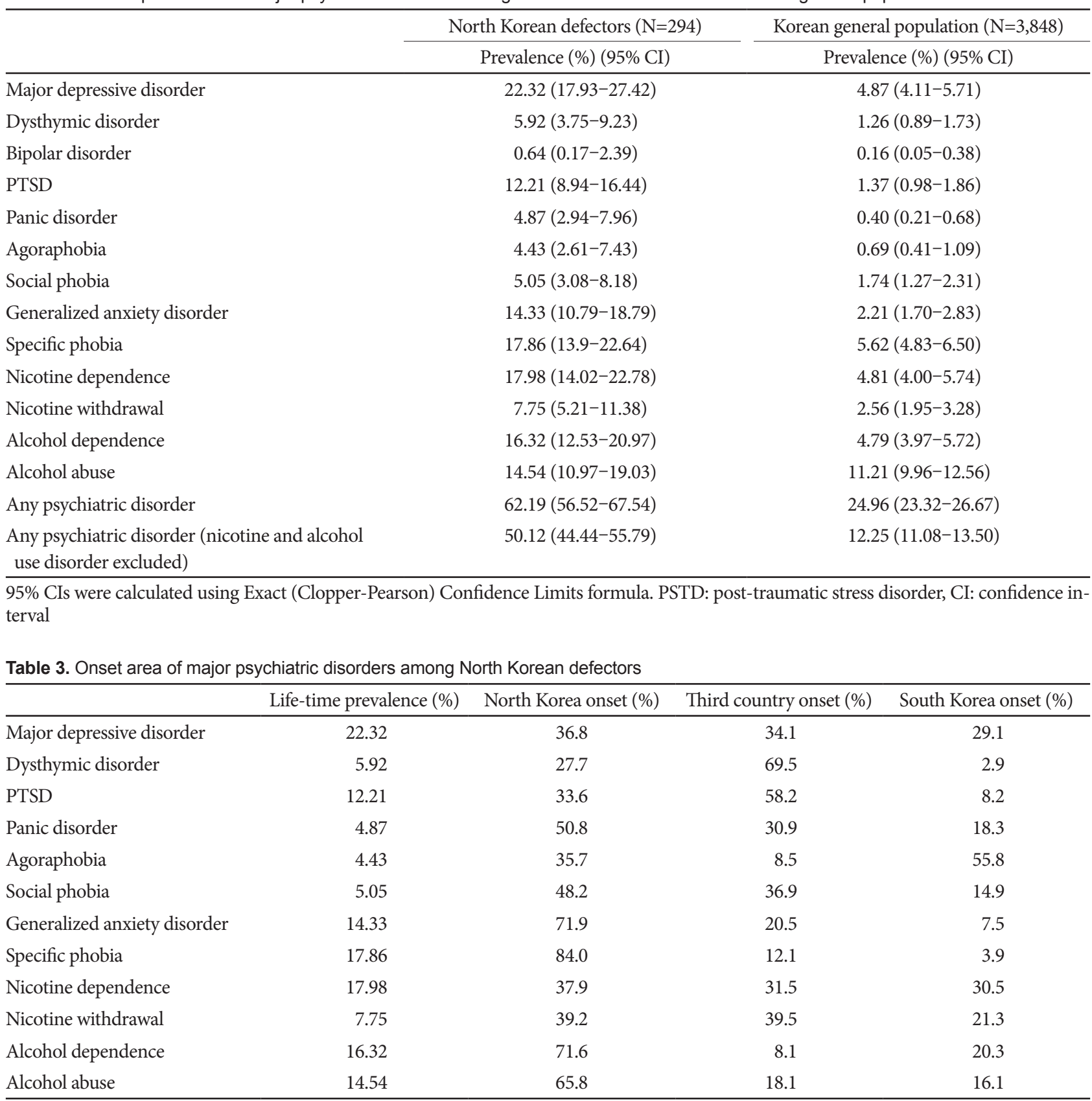

PSTD: post-traumatic stress disorder

opment of MDD. The stress factors including cultural differences, political tensions between the two countries and financial difficulties are suggested as factors that probably affected their depression in South Korea. ${ }^{35,36}$

In the case of PTSD and dysthymic disorder, the prevalence rates were higher in North Korean defectors than in the general Korean population. Considering a PTSD prevalence rate of $9 \%$ (CI 8-10\%) among the world's refugees who settled down in seven countries including the United States and European countries, ${ }^{5}$ the North Korean defectors were more vul- nerable to develop PTSD. Also, most of the participants who were diagnosed with PTSD or dysthymic disorder reported that their onset in a third country and North Korea while only few reported their psychiatric distress originating in South Korea. This higher likelihood of PTSD and dysthymic disorder developing in the third country and North Korea demonstrated that the fear of repatriation to North Korea, human trafficking or unfair discriminations affected their symptoms. ${ }^{9}$

Moreover, the prevalence of most anxiety disorders including panic disorder, social phobia, GAD, and specific phobia 
were higher among North Korean defectors than in the general South Korean populations. Its onset areas were mostly North Korea followed by a third country, which was probably related to their social background, and suggested a target of suspicion in their society under political surveillance. ${ }^{37}$ On the other hand, more than half of those who were diagnosed with agoraphobia reported its onset in South Korea. Previous studies suggested that perceived discrimination and acculturative stress affected their mental health. Since many of North Korean defectors in South Korea experienced discrimination and reported difficulties learning and adapting cultures, and the sample of this study involved those who recently settled down in South Korea, those factors might be related to high prevalence of agoraphobia and the maximum onset in South Korea. The onset age of agoraphobia is on average 30 years, known to be later than other anxiety disorders. ${ }^{38}$ North Korean defectors having experienced anxiety, panic, and PTSD in North Korea and third countries may develop agoraphobia after having settled in South Korea. Loneliness, social isolation and cultural difference may also affect the onset of agoraphobia in South Korea.

Although preliminary, differences existed between North Korean defectors and general Korean population in the lifetime prevalence of substance-related disorders including nicotine dependence and withdrawal, and alcohol dependence compared to other psychiatric disorders. This result is similar to a previous study reporting the high rate of alcohol use disorder in North Korean defectors ${ }^{15,39}$ and the data showing that $42.09 \%$ of men aged above 15 years smoked tobacco in North Korea daily. ${ }^{40}$ However, the onset area was not significantly different in nicotine dependence and withdrawal while, interestingly, alcohol dependence and abuse were mostly triggered in North Korea. Considering that North Korea is geographically close to China where drinking is a traditional part of life and strong liquor is common, ${ }^{41}$ the drinking pattern in North Korea might be affected. Moreover, since acculturation stress is related to drinking challenges, it is highly recommended to screen and assist those who have related problems among North Korean defectors residing in South Korea.

Several study limitations need to be discussed. First, since the sampling method was not random due to difficulties of approach and collection (e.g., the defectors' political and social security issues), the sample might not be representative of all the defector population. Also, the recall of symptoms experienced in the distant past might be inaccurate and biased due to issues related to memory. Notwithstanding these limitations, this study is meaningful as it is the first study to examine the prevalence of psychiatric disorders among North Korean defectors using the standardized diagnostic tool and compare the results with the general South Korean population.
The comparison between North Korean defectors and the general South Korean population suggests that the prevalence rates of major psychiatric disorders are higher among North Korean defectors. Also, this study informs the actual state of North Korean defectors' psychiatric disorders and offers useful resources for mental health policy makers underscoring the need for government intervention and support.

\section{Acknowledgments}

This work was supported by the Korea Healthcare Technology R\&D project, Ministry of Health and Welfare, Republic of Korea (HM15C1072).

The data that support the findings of this study are available from Korean National Center for Mental Health but restrictions apply to the availability of these data, which were still under ongoing study. But the data may be publicly available in the near future. Data are however available from the authors upon reasonable request and with permission of Korean National Center for Mental Health.

\section{Conflicts of Interest}

The authors have no potential conflicts of interest to disclose.

\section{Author Contributions}

Conceptualization: Jin Pyo Hong, Subin Park, Hye In Chang. Data curation: Ji Hyun An, Hyo Chul Lee, M Lee. Formal analysis: Kyoung Eun Lee, Ji Hyun An. Funding acquisition: Jin Pyo Hong. Investigation: Kyoung Run Lee, Minji Lee, Hyo Chul Lee. Methodology: Jin Pyo Hong, Eye In Chang. Project administration: Jin Pyo Hong, Hyo Chul Lee. Resources: Jin Pyo Hong. Software: Da Eun Kim. Supervision: Jin Pyo Hong. Validation: Jin Pyo Hong. Visualization: Kyoung Eun Lee. Writing—original draft: Kyoung Eun Lee, Carolyn Seungyoun Moon. Writing_-review \& editing: Subin Park, Jin Pyo Hong.

\section{ORCID iDs}

Kyoung Eun Lee

Carolyn Seungyoun Moon

Ji Hyun An

Hyo Chul Lee

Da Eun Kim

Subin Park

Minji Lee

Hye In Chang

Jin Pyo Hong

https://orcid.org/0000-0002-4980-796X https://orcid.org/0000-0001-8370-3745 https://orcid.org/0000-0002-1628-9617 https://orcid.org/0000-0002-9259-1949 https://orcid.org/0000-0001-6844-6998 https://orcid.org/0000-0002-4623-9899 https://orcid.org/0000-0001-6262-8627 https://orcid.org/0000-0001-8632-4978 https://orcid.org/0000-0001-5384-2605

\section{REFERENCES}

1. Kim SY. A study on settlement of North Korean defectors and supporting policies. J North-East Asian Stud 2009;50:231-250.

2. Unification Ministry of Korea. North Korean Defectors Government Policy. Seoul: Unification Ministry of Korea, Department of Settlement Support; 2017.

3. Jeon WT. Review of adaptation and mental health of refugees and perspectives and counterplots in Korean reunification process. J Korean Neuropsychiatr Assoc 1997;36:3-18.

4. Hollifield M, Warner TD, Lian N, Krakow B, Jenkins JH, Kesler J, et al. Measuring trauma and health status in refugees: a critical review. JAMA 2002;288:611-621.

5. Fazel M, Wheeler J, Danesh J. Prevalence of serious mental disorder in 7000 refugees resettled in western countries: a systematic review. Lancet 2005;365:1309-1314.

6. Jeon WT, Yu SE, Cho YA, Eom JS. Traumatic experiences and mental health of north korean refugees in South Korea. Psychiatry Investig 2008; 5:213-220. 
7. Lee Y, Lee MK, Chun KH, Lee YK, Yoon SJ. Trauma experience of North Korean refugees in China. Am J Prev Med 2001;20:225-229.

8. Jeon W, Hong C, Lee C, Kim DK, Han M, Min S. Correlation between traumatic events and posttraumatic stress disorder among North Korean defectors in South Korea. J Trauma Stress 2005;18:147-154.

9. Lee Y, Lee M, Park S. Mental health status of North Korean refugees in South Korea and risk and protective factors: a 10-year review of the literature. Eur J Psychotraumatol 2017;8:1369833.

10. Kim HH, Lee YJ, Kim HK, Kim JE, Kim SJ, Bae SM, et al. Prevalence and correlates of psychiatric symptoms in north korean defectors. Psychiatry Investig 2011;8:179-185.

11. Lee YJ, Jun JY, Park J, Kim S, Gwak AR, Lee SH, et al. Effects of Psychiatric Symptoms on Attention in North Korean Refugees. Psychiatry Investig 2016;13:480-487.

12. Kim J, Choi Y, Chae J. North Korean Defectors' depression through the CES-D and the Rorschach test. Korean J Cult Soc Issues 2008;14:41-61.

13. Shin HY, Lee H, Park SM. Mental health and its associated factors among North Korean defectors living in South Korea. Asia Pac J Public Health 2016;28:592-600.

14. Nam B, Kim JY, DeVylder JE, Song A. Family functioning, resilience, and depression among North Korean refugees. Psychiatry Res 2016;245: 451-457.

15. Jeon BH, Kim MD, Hong SC, Kim NR, Lee CI, Kwak YS, et al. Prevalence and correlates of depressive symptoms among North Korean defectors living in South Korea for more than one year. Psychiatry Investig 2009;6:122-130.

16. Ahn S, Jon D, Hong H, Jung M, Kim H, Hong N. Mental health of North Korean refugees: depression, anxiety and mental health service need. J Korean Assoc Soc Psychiatry 2012;17:83-88.

17. Kim S, Kim H, Kim J, Cho S, Lee Y. Relationship between physical illness and depression in North Korean defectors. Korean J Psychosom Med 2011;19:20-27.

18. Lee K, Kim Y, Yang M, Choi Y, Ko E, Lee G, et al. A study on the adaptation and depression of North Korean defectors to the South Korean society. Ewha J Nurs Sci 2007;41:14-22.

19. Choi SK, Min SJ, Cho MS, Joung H, Park SM. Anxiety and depression among North Korean young defectors in South Korea and their association with health-related quality of life. Yonsei Med J 2011;52:502-509.

20. Chang M, Son E. Complex PTSD symptoms and psychological problems of the North Korean defectors. Korean J Health Psychol 2014;19: 973-999.

21. Kim Y. Predictors for mental health problems among young North Korean refugees in South Korea. Contemp Soc Multiculture 2013;3:264285.

22. Yu SE, Jeon WT. Mental health of North Korean refugees in protective facilities in China. Psychiatry Investig 2008;5:70-77.

23. Kim Y, Jeon W, Cho Y. A study on the prevalence and the influencing factors of the mental health problems among recent migrant North Koreans: a focus on 2007 entrants. Unification Policy Res 2010;19:141-174.
24. Kim H. Difference on complex PTSD and PTSD symptoms according to types of traumatic events in North Korean refugees. Korean J Counsel Psychother 2012;31:1003-1022.

25. Cho MJ, Chang SM, Lee YM, Bae A, Ahn JH, Son J, et al. Prevalence of DSM-IV major mental disorders among Korean adults: a 2006 National Epidemiologic Survey (KECA-R). Asian J Psychiatr 2010;3:26-30.

26. Alonso J, Angermeyer MC, Bernert S, Bruffaerts R, Brugha TS, Bryson $\mathrm{H}$, et al. Sampling and methods of the European Study of the Epidemiology of Mental Disorders (ESEMeD) project. Acta Psychiatr Scand Suppl 2004;(420):8-20.

27. Department of Health and Welfare. The Survey of Mental Disorders in Korea. Seoul: Department of Health and Welfare; 2017.

28. Robins LN, Wing J, Wittchen HU, Helzer JE, Babor TF, Burke J, et al. The Composite International Diagnostic Interview. An epidemiologic Instrument suitable for use in conjunction with different diagnostic systems and in different cultures. Arch Gen Psychiatry 1988;45:1069-1077.

29. Cho MJ, Hahm BJ, Suh DW, Hong JP, Bae JN, Kim JK, et al. Development of a Korean version of the composite international diagnostic interview (K-CIDI). J Korean Neuropsychiatr Assoc 2002;41:123-127.

30. Ha JH. Understanding of emotional communication pattern of North Korea defectors. Konkuk Univ Humanit Res Inst 2012;5:303-329.

31. Lee T, Ahn MH, Jun JY, Han JM, Lee SH, Hahm BJ, et al. Development of North Korean version of the composite international diagnostic interview. J Korean Neuropsychiatr Assoc 2015;54:228-235.

32. Statistics Korea. Korea National Statistical Office 2016. Available at: http://kostat.go.kr/portal/korea/kor_nw/2/2/2/index. board?bmode=read\&aSeq=358170. Accessed May 21, 2017.

33. Agresti A, Coull BA. Approximate is better than "exact" for interval estimation of binomial proportions. Am Stat 1998;52:119-126.

34. Porter M, Haslam N. Predisplacement and postdisplacement factors associated with mental health of refugees and internally displaced persons: a meta-analysis. JAMA 2005;294:602-612.

35. Min SK. Divided countries, divided mind 1: psycho-social issues in adaptation problems of North Korean defectors. Psychiatry Investig 2008; 5:1-13.

36. Min SK, Jeon WT, Yoon DR. Life and development of adolescents in North Korea. J Korean Neuropsychiatr Assoc 1999;38:1047-1062.

37. Yoo HR. Analysis of the North Korean system trauma anxiety through North Korean refugees. Korean J Christian Counsel 2014;25:117-155.

38. Michael T, Zetsche U, Margraf J. Epidemiology of anxiety disorders. Epidemiol Psychopharmacol 2007;6:136-142.

39. Jeon WT. Survey results of adaptation and life of North Korean defectors in South Korea, 2001. Korean Unification Stud 2003;7:155-208.

40. The Tobacco Atlas. Country Fact Sheet: DPR Korea. World Lung Foundation. Available at: https://tobaccoatlas.org/wp-content/uploads/pdf/ dpr-korea-country-facts.pdf. Accessed May 21, 2017.

41. Cochrane J, Chen H, Conigrave KM, Hao W. Alcohol use in China. Alcohol Alcohol 2003;38:537-542. 